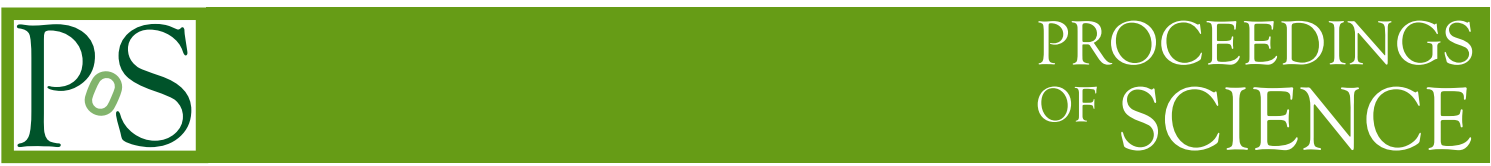

\title{
Recent results on hard and rare probes from ATLAS
}

\section{Mariusz Przybycien ${ }^{\dagger}, *$ on behalf of the ATLAS Collaboration}

AGH University of Science and Technology,

Faculty of Physics and Applied Computer Science,

Al. Mickiewicza 30, 30-055, Krakow, Poland

E-mail: mariusz.przybycien@agh.edu.pl

Hard probes are indispensable tools to study the hot and dense hadronic matter created in ultrarelativistic heavy-ion collisions. They may either strongly interact with the surrounding medium, like partons being proxies of jets, or both their production rates and their passing through the medium might be unaffected by the final-state effects, as it is in the case of electroweak bosons. So they can provide information on both the properties of the final-state medium and the characteristics of the initial state. Recent ATLAS results on jets and electroweak bosons production in $\mathrm{Pb}+\mathrm{Pb}$ and $p p$ collisions are reviewed. On the other side, in ultra-peripheral collisions of heavy-ions, the two-photon interactions are studied. A recent study of the light-by-light scattering process focussing on a possible observation of the axion-like particle is briefly discussed, and new limits are obtained.

The Eighth Annual Conference on Large Hadron Collider Physics-LHCP2020

25-30 May, 2020

online

\footnotetext{
${ }^{*}$ Speaker

$\dagger$ This work was partly supported by the National Science Centre of Poland under grant number UMO2016/23/B/ST2/01409, by the AGH UST statutory task No. 11.11.220.01/4 within subsidy of the Ministry of Science and Higher Education and by PL-GRID infrastructure.
} 


\section{Introduction}

The Quark Gluon Plasma (QGP), a new state of matter produced in ultrarelativistic heavyion (HI) collisions, exists only for a short time, $t \sim 10 \mathrm{fm} / c \sim 10^{-23} \mathrm{~s}$, after a collision. Therefore it can not be studied with external probes, but rather a probe from inside the collision region is needed. Hard probes are produced early in a HI collision, in a process for which the cross section is not changed by the presence of the strongly interacting medium, i.e. can be calculated within pQCD. Passing through the medium, hard probes interact weakly or strongly with it, providing information on its properties. In these proceedings, recent studies of the QGP performed at the ATLAS [1] experiment, in terms of hard probes such as jets and electroweak bosons, are presented. Also rare processes such as the light-by-light (LbyL) scattering are briefly discussed. All results presented here are obtained using $\mathrm{Pb}+\mathrm{Pb}$ and $p p$ data collected at $\sqrt{s_{\mathrm{NN}}}=5.02 \mathrm{TeV}$.

\section{Recent ATLAS measurements}

It is well known that the high transverse momentum partons, produced in hard scattering processes and propagating through the medium of strongly interacting nuclear matter, lose their energy, resulting in the phenomenon of "jet quenching". Fragmentation of jets into charged particles in HI collisions can provide information about the mechanism of jet quenching in the QGP.

ATLAS has recently measured the angular distribution of charged particles around the jet axis in $\mathrm{Pb}+\mathrm{Pb}$ and $p p$ collisions [2]. The measurements are performed for jets reconstructed with the anti- $k_{t}$ algorithm with radius parameter $R=0.4$ and are extended to an angular distance of $r=0.8$ from the jet axis. The results presented here are quantified using the following two observables:

$$
D\left(p_{\mathrm{T}}, r\right)=\frac{1}{N_{\mathrm{jet}}} \frac{1}{2 \pi r d r} \frac{d n_{\mathrm{ch}}\left(p_{\mathrm{T}}, r\right)}{d p_{\mathrm{T}}} \quad R_{D\left(p_{\mathrm{T}}, r\right)}=\frac{D\left(p_{\mathrm{T}}, r\right)_{\mathrm{Pb}+\mathrm{Pb}}}{D\left(p_{\mathrm{T}}, r\right)_{p p}}
$$

where $N_{\text {jet }}$ is the number of jets in consideration and $n_{\mathrm{ch}}\left(p_{\mathrm{T}}, r\right)$ is the number of charged particles with a given $p_{\mathrm{T}}$ at a distance $r$ from the jet axis. The ratios of the charged-particle yields, $R_{D\left(p_{\mathrm{T}}, r\right)}$, measured in $\mathrm{Pb}+\mathrm{Pb}$ and $p p$ collisions, quantify the modifications of the yields due to the QGP medium. The above quantities have been measured in several ranges of the jet transverse momentum, $p_{\mathrm{T}}^{\mathrm{jet}}$, and for several centrality intervals. The results for the most central events and for jets with $126 \mathrm{GeV}<p_{\mathrm{T}}^{\text {jet }}<158 \mathrm{GeV}$ are shown in Figure 1 . The $D\left(p_{\mathrm{T}}, r\right)$ distributions decrease as a function of $r$. The rate of fall-off increases sharply for higher $p_{\mathrm{T}}$ particles, with most of these being concentrated near the jet axis. The distributions exhibit a difference in shape between $\mathrm{Pb}+\mathrm{Pb}$ and $p p$ collisions, with the $\mathrm{Pb}+\mathrm{Pb}$ distributions being broader at low $p_{\mathrm{T}}\left(p_{\mathrm{T}}<4 \mathrm{GeV}\right)$ and narrower at high $p_{\mathrm{T}}\left(p_{\mathrm{T}}>4 \mathrm{GeV}\right)$ in $0-10 \%$ central collisions. This modification is centrality dependent and is smaller for peripheral $\mathrm{Pb}+\mathrm{Pb}$ collisions (not shown). The $R_{D\left(p_{\mathrm{T}}, r\right)}$ distributions for charged particles with $p_{\mathrm{T}}<4 \mathrm{GeV}$ are above unity and grow with increasing angular separation up to $r \sim 0.3$, showing weak to no dependence on $r$ in the interval $0.3<r<0.6$ followed by a small decrease in the enhancement for $0.6<r<0.8$. For charged particles with $p_{\mathrm{T}}>4 \mathrm{GeV}$, a suppression is observed and the size of the modification increases with increasing $r$ for $0.05<r<0.3$ with no $r$ dependence for $r>0.3$. For all charged-particle $p_{\mathrm{T}}$ values, the $R_{D\left(p_{\mathrm{T}}, r\right)}$ is greater than or equal to unity for $r<0.05$. For $0.1<r<0.25$, a statistically significant trend of increasing $R_{D\left(p_{\mathrm{T}}, r\right)}$ with 

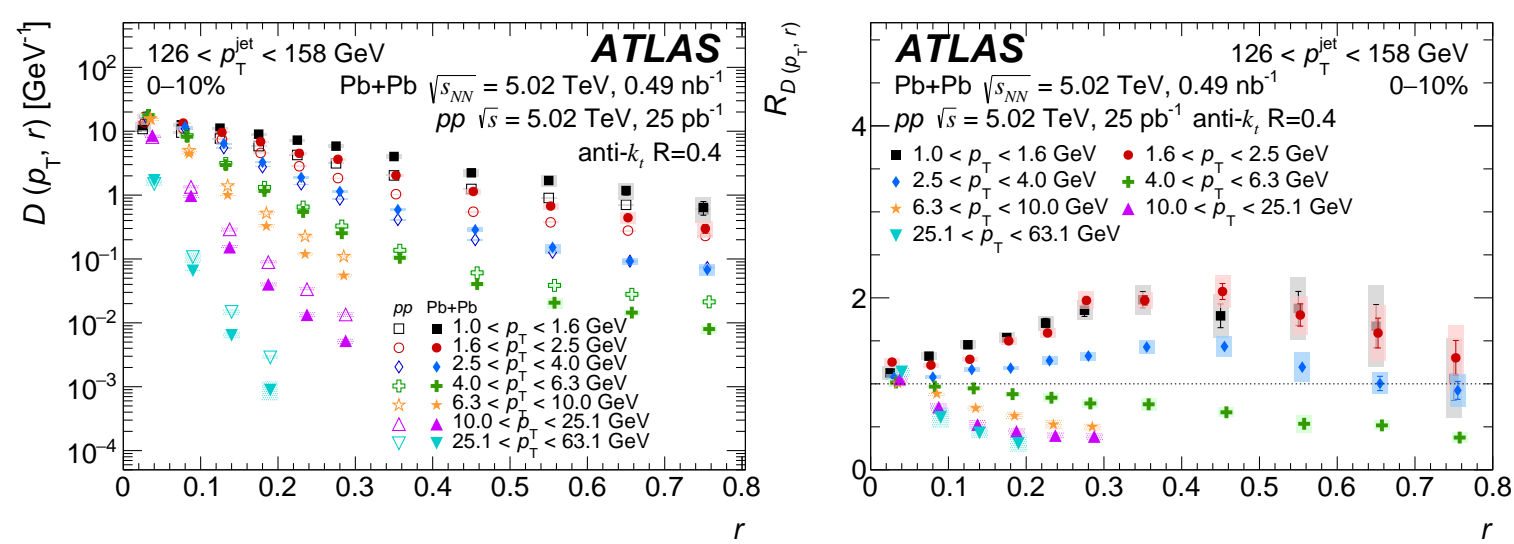

Figure 1: (left) The $D\left(p_{\mathrm{T}}, r\right)$ distributions in $p p$ (open symbols) and $\mathrm{Pb}+\mathrm{Pb}$ (closed symbols), and (right) the ratios $R_{D\left(p_{\mathrm{T}}, r\right)}$, both as functions of the angular distance $r$ for $p_{\mathrm{T}}^{\text {jet }}$ of 126 to $158 \mathrm{GeV}$ and for the most central events, $0-10 \%$. The symbols represent different track $p_{\mathrm{T}}$ ranges. The vertical bars on the data points indicate statistical uncertainties, while the shaded boxes indicate systematic uncertainties. Taken from Ref. [2].

increasing $p_{\mathrm{T}}^{\mathrm{jet}}$ is observed for low- $p_{\mathrm{T}}$ particles. No significant $p_{\mathrm{T}}^{\text {jet }}$ dependence is seen for particles with $p_{\mathrm{T}}>4 \mathrm{GeV}$.

ATLAS has also studied [3] the substructure of jets in $\mathrm{Pb}+\mathrm{Pb}$ collisions using a large radius parameter, $R=1.0$. The $R=1.0$ jets are clustered from small-radius, $R=0.2$, anti- $k_{t}$ jets with $p_{\mathrm{T}}>35 \mathrm{GeV}$ reconstructed using the standard ATLAS HI jet reconstruction algorithm. This procedure limits the impact of the underlying event (UE) on the measurement but also does not allow recovering the energy transferred outside the $R=0.2$ sub-jets via jet quenching. Therefore, this measurement studies the hard parton splittings. The anti- $k_{t}$ jet algorithm is used to re-cluster $R=1.0$ jet constituents to obtain the $k_{t}$ splitting scales. The splitting scales are determined by clustering objects according to their distance from each other with the following distance definition $d_{i j}=\min \left(p_{\mathrm{T}, i}^{2}, p_{\mathrm{T}, j}^{2}\right) \cdot \Delta R_{i j}^{2}$, with $\Delta R_{i j}=\sqrt{\Delta \phi_{i j}^{2}+\Delta y_{i j}^{2}}$, where input objects are labeled with an index corresponding to the $i$-th and $j$-th momentum in the list of inputs, for all possible permutations of $i$ and $j$ in the given clustering step. We present the measurement of the splitting scale $\sqrt{d_{12}}$, where indices 1 and 2 refer to the two jets before the final clustering step. For large-radius jets with only a single sub-jet, $\sqrt{d_{12}}$ is defined to be 0 . The jet yield measured in $\mathrm{Pb}+\mathrm{Pb}$ collisions is compared to that measured in $p p$ collisions using the nuclear modification factor, $R_{\mathrm{AA}}$, measured as a function of jet $p_{\mathrm{T}}$ and $\sqrt{d_{12}}$ in different $\mathrm{Pb}+\mathrm{Pb}$ centrality intervals, and defined as:

$$
R_{\mathrm{AA}}=\frac{1}{N_{\mathrm{evt}}} \frac{1}{\left\langle T_{\mathrm{AA}}\right\rangle}\left(\left.\frac{d^{3} N_{\mathrm{jet}}}{d p_{\mathrm{T}} d \sqrt{d_{12}} d y}\right|_{\mathrm{cent}}\right) / \frac{d^{3} \sigma_{\mathrm{jet}}^{p p}}{d p_{\mathrm{T}} d \sqrt{d_{12}} d y} .
$$

The $R_{\mathrm{AA}}$ evaluated as a function of jet $p_{\mathrm{T}}$ in five centrality intervals is shown in Figure 2 (left). A clear suppression of the inclusive large-radius jet production in central $\mathrm{Pb}+\mathrm{Pb}$ collisions with respect to $p p$ collisions can be seen. In $0-10 \%$ central collisions $R_{\mathrm{AA}}$ reaches a value of approximately 0.5 at $p_{\mathrm{T}}=200 \mathrm{GeV}$ while growing slowly with increasing jet $p_{\mathrm{T}}$. It stays below 0.6 for jets with $p_{\mathrm{T}}$ up to $600 \mathrm{GeV}$. The $R_{\mathrm{AA}}$ evaluated as a function of $\sqrt{d_{12}}$ is shown in Figure 2 (middle). The $R_{\mathrm{AA}}$ values for large-radius jets with a single sub-jet are significantly different compared to the $R_{\mathrm{AA}}$ for large-radius jets with a more complex substructure with a non-zero $\sqrt{d_{12}}$. The $R_{\mathrm{AA}}$ sharply decreases with increasing $\sqrt{d_{12}}$ for small values of the splitting scale followed by some flattening 

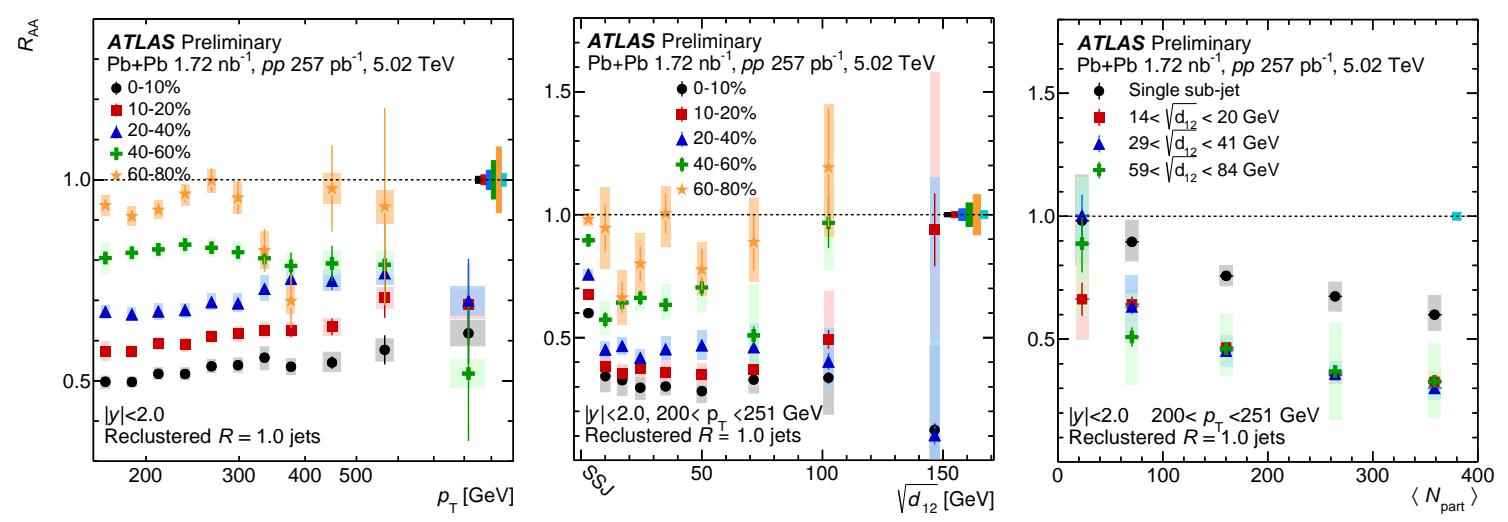

Figure 2: The values of $R_{\mathrm{AA}}$ for inclusive $R=1.0$ jets as a function of (left) jet $p_{\mathrm{T}}$ in five centrality intervals, (middle) $k_{t}$ splitting scale, $\sqrt{d_{12}}$, in five centrality intervals and for $200 \mathrm{GeV}<p_{\mathrm{T}}^{\text {jet }}<251 \mathrm{GeV}$, and (right) $\left\langle N_{\text {part }}\right\rangle$ for $200 \mathrm{GeV}<p_{\mathrm{T}}^{\text {jet }}<251 \mathrm{GeV}$ and different intervals of $\sqrt{d_{12}}$. The vertical bars on the data points indicate statistical uncertainties, while the shaded boxes indicate systematic uncertainties. The systematic uncertainties on yields also include the uncertainty due to the luminosity and the nuclear overlap function $\left\langle T_{\mathrm{AA}}\right\rangle$, which is correlated for all the data points. Taken from Ref. [3].

for larger $\sqrt{d_{12}}$. The $R_{\mathrm{AA}}$ evaluated as a function of $\left\langle N_{\text {part }}\right\rangle$ is shown in Figure 2 (right) for four $\sqrt{d_{12}}$ intervals. A continuous increase of the suppression with increasing centrality is observed. The jets with a single sub-jet are less suppressed with respect to those with higher sub-jet multiplicity.

Due to lack of space, for the discussion of the recent ATLAS results on $W^{ \pm}$and $Z$ bosons production [4-6], presented at the conference, the interested reader is referred to Ref. [7]. However, it is worth mentioning here, following Ref. [8], that when reducing the inelastic nucleon-nucleon cross section in the Glauber model calculations, one can obtain much better agreement between the ATLAS results and the predictions based on nPDFs. This reduction of the inelastic cross section is motivated by gluon shadowing and saturation phenomena at small Bjorken- $x$, which might be enhanced in HI collisions.

The electromagnetic fields produced by the colliding $\mathrm{Pb}$ nuclei can be treated as beams of quasi-real photons with a small virtuality. In ultra-peripheral HI collisions, the strong interaction between colliding nuclei can be neglected, and the collision can be regarded as interaction of photons. ATLAS has already studied [9] the LbyL collision process, $\gamma \gamma \rightarrow \gamma \gamma$, proceeding at the lowest order in QED via virtual one-loop box diagrams involving charged particles. The measurements have been extended recently, using full available data statistics [10]. In Figure 3 (left) the diphoton invariant mass distribution is shown, for events fulfilling the following requirements applied on the two final-state photons: $\left|\eta^{\gamma}\right|<2.4, p_{\mathrm{T}}^{\gamma}>2.5 \mathrm{GeV}, p_{\mathrm{T}}^{\gamma \gamma}<1 \mathrm{GeV}$, and the two photons should be back-to-back within an accoplanarity $\mathrm{A}_{\phi}<0.01$. Any particle directly coupling to photons could be produced in an $s$-channel process in $\gamma \gamma$ collisions, leading to a resonance peak in the invariant mass spectrum. The measured diphoton invariant mass distribution is used to search for axion-like particles (ALP) and set new exclusion limits on their production in the $\gamma \gamma \rightarrow a \rightarrow \gamma \gamma$ reaction. Assuming a 100\% ALP decay branching fraction into photons, the derived constraints on the ALP mass and its coupling to photons, $\left(1 / \Lambda_{a}\right)$, are compared in Figure 3 (right) with those obtained from other measurements. These results provide, to this date, the most stringent constraints in the search for ALP signals in the $6-100 \mathrm{GeV}$ mass range. 

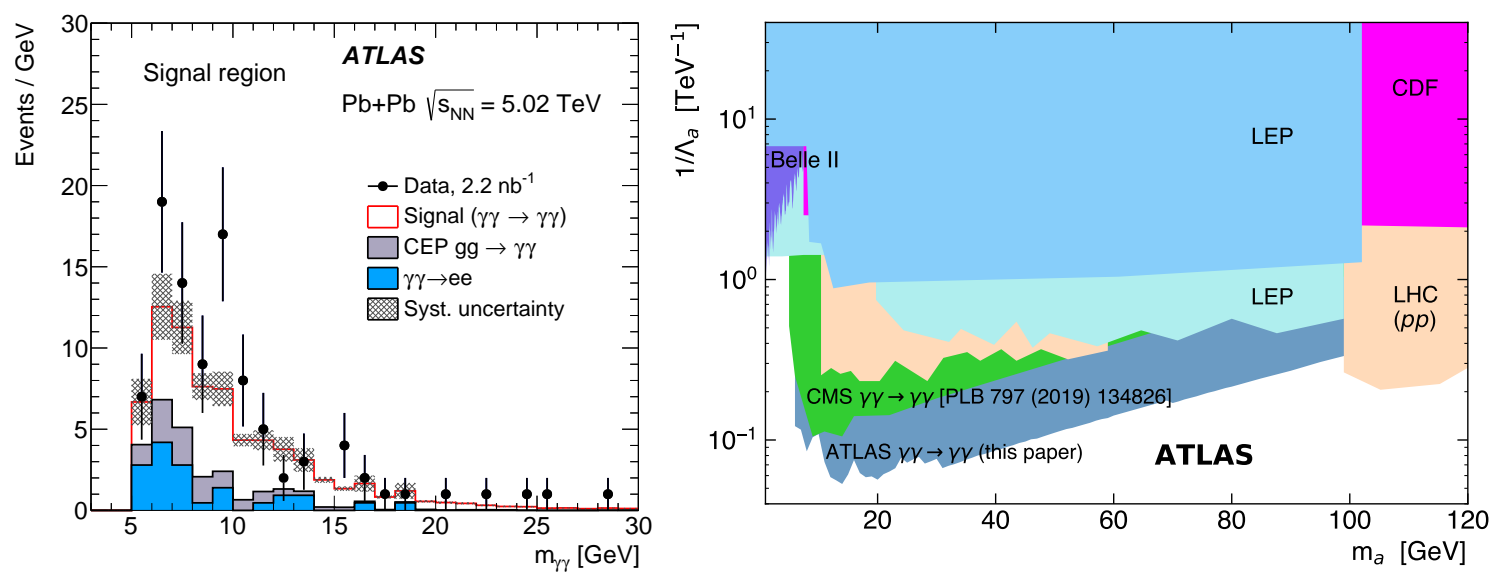

Figure 3: (left) Invariant mass of the diphoton candidates. (right) Compilation of exclusion limits at $95 \%$ confidence level in the ALP-photon coupling $\left(1 / \Lambda_{a}\right)$ versus ALP mass $\left(m_{a}\right)$ plane obtained by different experiments. The existing limits are compared to the limits extracted from this measurement. The exclusion limits denoted as "LHC" are based on pp collision data from ATLAS and CMS. Taken from Ref. [10].

\section{References}

[1] ATLAS Collaboration, The ATLAS Experiment at the CERN LHC, JINST 3 (2008) S08003.

[2] ATLAS Collaboration, Measurement of angular and momentum distributions of charged particles within and around jets in $\mathrm{Pb}+\mathrm{Pb}$ and pp collisions at $\sqrt{s_{\mathrm{NN}}}=5.02 \mathrm{TeV}$ with the ATLAS detector, Phys. Rev. C 100, 064901 (2019), arXiv:1908.05264 [nucl-ex].

[3] ATLAS Collaboration, Measurement of suppression of large-radius jets and its dependence on substructure in $\mathrm{Pb}+\mathrm{Pb}$ at $5.02 \mathrm{TeV}$ with the ATLAS detector, ATLAS-CONF-2019-056.

[4] ATLAS Collaboration, Measurement of $W^{ \pm}$boson production in $\mathrm{Pb}+\mathrm{Pb}$ collisions at $\sqrt{s_{\mathrm{NN}}}=5.02 \mathrm{TeV}$ with the ATLAS detector, Eur. Phys. J. C 79, 935 (2019), arXiv:1907.10414 [nucl-ex].

[5] ATLAS Collaboration, $Z$ boson production in $P b+P b$ collisions at $\sqrt{s_{\mathrm{NN}}}=5.02 \mathrm{TeV}$ measured by the ATLAS experiment, Phys. Lett. B 802, 135262 (2020), arXiv:1910.13396 [nucl-ex].

[6] ATLAS Collaboration, Measurements of $W$ and $Z$ boson production in pp collisions at $\sqrt{s}=5.02 \mathrm{TeV}$ with the ATLAS detector, Eur. Phys. J. C 79, 128 (2019), arXiv:1810.08424 [hep-ex].

[7] M. Przybycien, Production of electroweak bosons in $\mathrm{Pb}+\mathrm{Pb}, \mathrm{p}+\mathrm{Pb}$ and pp collisions with the ATLAS detector, European Physical Society Conference on High Energy Physics (EPS HEP 2019), 10 - 17 July 2019, Ghent, Belgium, PoS(EPS-HEP2019)300.

[8] K.J. Eskola, I. Helenius, M. Kuha, H. Paukkunen, Shadowing in inelastic nucleon-nucleon cross section?, arXiv:2003.11856 [hep-ph].

[9] ATLAS Collaboration, Evidence for light-by-light scattering in heavy-ion collisions with the ATLAS detector at the LHC, Nature Phys. 13 (2017) 852, arXiv:1702.01625 [hep-ex].

[10] ATLAS Collaboration, Measurement of light-by-light scattering and search for axion-like particles with $2.2 n b^{-1}$ of $\mathrm{Pb}+\mathrm{Pb}$ data with the ATLAS detector, arXiv:2008.05355 [hep-ex]. 